\title{
Generalized Guidance Scheme for Low-Thrust Orbit Transfer
}

\author{
Henzeh Leeghim, ${ }^{1}$ Dong-Hyun Cho, ${ }^{2}$ Su-Jang Jo, ${ }^{3}$ and Donghoon Kim ${ }^{4}$ \\ ${ }^{1}$ Department of Aerospace Engineering, Chosun University, Gwangju 501-759, Republic of Korea \\ ${ }^{2}$ Future Convergence Research Division, Korea Aerospace Research Institute, Daejeon 305-806, Republic of Korea \\ ${ }^{3}$ Department of Aerospace Engineering, KAIST, Daejeon 305-701, Republic of Korea \\ ${ }^{4}$ Texas A\&M University, College Station, TX 77843-3141, USA
}

Correspondence should be addressed to Donghoon Kim; aerospace38@gmail.com

Received 21 May 2014; Accepted 21 August 2014; Published 15 September 2014

Academic Editor: Guido Maione

Copyright (C) 2014 Henzeh Leeghim et al. This is an open access article distributed under the Creative Commons Attribution License, which permits unrestricted use, distribution, and reproduction in any medium, provided the original work is properly cited.

\begin{abstract}
The authors present an orbital guidance scheme for the satellite with an electrical propulsion system using a Lyapunov feedback control. The construction of a Lyapunov candidate is based on orbital elements, which consist of angular momentum and eccentricity vectors. This approach performs orbit transfers between any two arbitrary elliptic or circular orbits without any singularity issues. These orbital elements uniquely describe a non degenerate Keplerian orbit. The authors improve the reliability of the existing Lyapunov orbital guidance scheme by considering the energy term. Additional improvement is achieved by adding the penalty function. Furthermore, it is shown that the final suggested approach is suitable for the satellite passing the earth's shadow area.
\end{abstract}

\section{Introduction}

Orbit transfer problems for a satellite, which has an electric propulsion system, are very interesting and difficult because of the low and continuous thrust of the electric propulsion system. There are various approaches to solve the orbit transfer problem. Some of the earliest work in this field has focused on finding optimal trajectories using either indirect or direct techniques or mixtures of them [1-4]. The orbit transfer duration of the satellite using an electric propulsion system, however, is quite long. It means that obtaining control profiles for the transfer orbit is extremely difficult for the onboard computer of a satellite to calculate. In addition, trajectory optimization methods typically determine an openloop steering program that may be difficult or impractical to store at the on-board computer. Therefore, orbital guidance schemes are demanded for interplanetary or small satellites [5].

There are two categories of the approach for orbital guidance. One category (see [6-8]) involves "blending" the instantaneously optimal thrust directions for changing each of the orbital elements during each of several phases of the orbit transfer. The second category (see [9-14]) is based on Lyapunov feedback controls, where a suitable Lyapunov function candidate must be defined by mission designers. Ilgen [9] has suggested a Lyapunov candidate is based on only five of the classical orbital elements. While the physical interpretations of the classical orbital elements are easy to visualize, this set of orbital elements often leads to singular equations as the eccentricity and the orbit inclination angle tend to zero. Chang et al. [13] have proposed a Lyapunov candidate is based on the orbital elements, which consist of angular momentum and eccentricity vectors. This approach helps to avoid the singular equations.

Moreover, small satellites on low earth orbits (LEOs) can be in the earth's shadow area, which is an important issue [15, 16]. Supplying electric powers to the satellite during the time when it is in the earth's shadow area is going to be difficult due to insufficient sun powers. Hence, the earth's shadow effect should be taken into account for guidance schemes for orbit transfers. This work studies an orbital guidance law of a satellite passing through the shadow area of the earth using an electric propulsion system for transferring orbits. Note that the Lyapunov feedback control law is the most promising technology for orbit transfers because of its simplicity. 


\section{Review of Orbital Dynamics}

In classical orbital mechanics, six orbital elements are usually used to describe motions of an orbiting body. These classical orbital elements, however, have some singularity issues when the eccentricity is 0 or the orbit inclination is $0^{\circ}$ or $180^{\circ}$. The equinoctial orbital elements are used to avoid singularity issues, but the state dynamics, by using calculus of variation, are very complex. In contrast with the orbital elements, a vector notation using the angular momentum and the eccentricity vectors is very simple, and the singularities can also be avoided. For this reason, the vector notation is utilized for this work.

Every nondegenerate Keplerian orbit can be uniquely described by the angular momentum and the eccentricity vectors. In general, six elements are required to describe the motion of the particle moving around the massive body. In this study, the final time is not specified and the injection point is free. That is, only five elements are required to solve the orbit transfer problem. Note that these two orthogonal vectors can be uniquely used to describe the initial and desired orbits.

From Figure 1, one can easily convert the orbital elements to the following vector notations:

$$
\begin{gathered}
\mathbf{h}=\sqrt{\mu a\left(1-e^{2}\right)}\left[R_{3}(w) R_{1}(i) R_{3}(\Omega)\right]^{T}[0,0,1]^{T}, \\
\mathbf{e}=\left[R_{3}(w) R_{1}(i) R_{3}(\Omega)\right]^{T}[e, 0,0]^{T},
\end{gathered}
$$

where $R_{i}(\cdot)$ is the rotational matrix for the ith Euler angle, $\mathbf{e}$ is the eccentricity vector, $\mathbf{h}$ is the angular momentum vector, $a$ is the semimajor axis, $\mu$ is the earth gravitational constant, and $e$ is the eccentricity. Because these vector notations do not consider a particle's position in orbits, additional parameters are required for rendezvous missions.

A satellite motion, which includes only the gravitational force from the earth, is considered and the governing equation is given by [17]

$$
\ddot{\mathbf{r}}=-\frac{\mu}{r^{3}} \mathbf{r}+\mathbf{a}_{T}
$$

where $\mathbf{r}$ is the position vectors of the spacecraft in Earthcentered inertial (ECI) coordinate frames and the thrust acceleration vector $\mathbf{a}_{T}$ in the ECI frame is described by [10]

$$
\mathbf{a}_{T}=-\frac{2 \eta P}{m g I_{\mathrm{SP}}} \widehat{\mathbf{u}}=a_{T} \widehat{\mathbf{u}},
$$

where $m$ is the mass of spacecraft, $P$ is the input power to the electric propulsion system, $\eta$ is the engine efficiency, $g$ is the earth gravitational acceleration, $I_{\mathrm{SP}}$ is the specific impulse, and $\widehat{\mathbf{u}}$ is the unit vector along the thrust direction. Note that the unit thrust vector direction is the control command and an acceleration vector due to perturbations is not considered. The mass flow rate due to the low-thrust engine is given by [10]

$$
\dot{m}=-\frac{2 \eta P}{\left(g I_{\mathrm{SP}}\right)^{2}}
$$

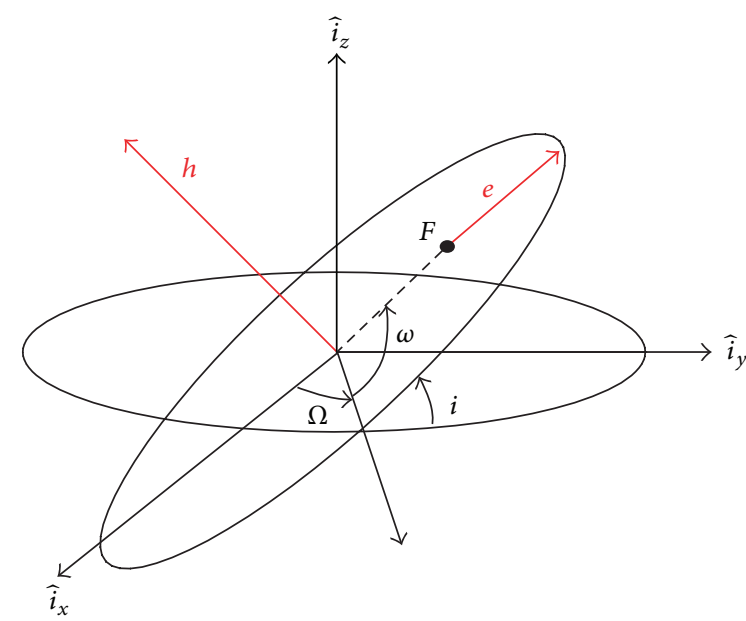

FIgURE 1: The conversion between classical orbital elements and vector notation.

The differential equations for $\mathbf{e}$ and $\mathbf{h}$ in (1) are expressed as

$$
\begin{gathered}
\dot{\mathbf{h}}=a_{T}[\widetilde{\mathbf{r}}] \widehat{\mathbf{u}}, \\
\dot{\mathbf{e}}=a_{T} \Gamma \widehat{\mathbf{u}},
\end{gathered}
$$

where $\Gamma$ is defined as

$$
\Gamma=\frac{1}{\mu}[\widetilde{\mathbf{v}}][\widetilde{\mathbf{r}}]
$$

and the notation $[\widetilde{\mathbf{d}}]$ represents

$$
[\tilde{\mathbf{d}}]=\left[\begin{array}{ccc}
0 & -d_{3} & d_{2} \\
d_{3} & 0 & -d_{1} \\
-d_{2} & d_{1} & 0
\end{array}\right],
$$

where $\mathbf{d} \equiv\left[d_{1}, d_{2}, d_{3}\right]^{T}$ is the generic variable.

\section{Previous Guidance Schemes}

There is much research about orbital guidance schemes for satellites moving around the earth or other planets. In these researches, the guidance scheme using the Lyapunov feedback control method is suitable for the satellites with an electric propulsion system. Because these satellites have very low thrust for the orbit transfer mission, the orbit transfer duration is quite long. That is, during the orbit transfer, the reliability of the convergence is a very important factor, and the reliability can be verified by using the Lyapunov feedback control.

Many researchers have explored the Lyapunov feedback control. For example, Vadali et al. [12] have used the Lyapunov feedback control for the earth capture mission. This method, however, is only applicable to two-dimensional orbit transfer missions because scalar terms for the Lyapunov feedback control are only considered. In contrast with this work, Chang et al. [13] have used the vector notation to consider threedimensional orbit transfer missions. In this section, this orbital guidance scheme is introduced. 
Consider a Lyapunov candidate

$$
\begin{aligned}
V= & \frac{1}{2}\left(\mathbf{e}-\mathbf{e}_{f}\right)^{T} W_{1}\left(\mathbf{e}-\mathbf{e}_{f}\right) \\
& +\frac{1}{2} \frac{\left(\mathbf{h}-\mathbf{h}_{f}\right)^{T} W_{2}\left(\mathbf{h}-\mathbf{h}_{f}\right)[\widetilde{\mathbf{r}}]}{\left\|\mathbf{h}_{f}\right\|},
\end{aligned}
$$

where $\mathbf{e}_{f}$ and $\mathbf{h}_{f}$ are the desired eccentricity and angular momentum vectors, respectively. Note that the angular momentum vector term is normalized because this value is very large. On the other hand, the eccentricity vector is not normalized to avoid the singularity as the eccentricity approaches zero. $W_{1}$ and $W_{2}$ are the positive definite gain matrices. Thus, the Lyapunov candidate always has positive values except when the satellite achieved the desired orbit state. Therefore, during the orbit transfer, the time derivative of the Lyapunov candidate must have negative values to let the Lyapunov candidate go to zero.

Using (5), the time derivative of the Lyapunov candidate in (8) is written as

$$
\frac{\mathrm{d} V}{\mathrm{~d} t}=\left[\left(\mathbf{e}-\mathbf{e}_{f}\right)^{T} W_{1} \Gamma+\frac{\left(\mathbf{h}-\mathbf{h}_{f}\right)^{T} W_{2}[\widetilde{\mathbf{r}}]}{\left\|\mathbf{h}_{f}\right\|}\right] a_{T} \widehat{\mathbf{u}},
$$

where $\widehat{\mathbf{u}}$ is the control parameter which is selected to make (9) negative as follows:

$$
\widehat{\mathbf{u}}=-\frac{\left[\left(\mathbf{e}-\mathbf{e}_{f}\right)^{T} W_{1} \Gamma+\left(\mathbf{h}-\mathbf{h}_{f}\right)^{T} W_{2}[\widetilde{\mathbf{r}}] /\left\|\mathbf{h}_{f}\right\|\right]^{T}}{\left\|\left(\mathbf{e}-\mathbf{e}_{f}\right)^{T} W_{1} \Gamma+\left(\mathbf{h}-\mathbf{h}_{f}\right)^{T} W_{2}[\widetilde{\mathbf{r}}] /\right\| \mathbf{h}_{f}\|\|} .
$$

Now, (9) is always negative except the following two cases: (i) the orbit reaches the desired orbit and (ii) the thrust magnitude is zero. Case (ii) will be discussed in Section 5.

\section{Improved Guidance Schemes}

The guidance scheme [13] performs well even though the orbit state has some singularity conditions. However, this guidance scheme also has some problems by using the vector notation. One of the problems is caused when large-angle maneuvers are performed. Because this orbital guidance scheme uses the vector control, the transition vector is varying during the orbit transfer as shown in Figure 2. This transition vector is not exactly passing the line between the initial and final angular momentum vectors. This phenomenon comes from the coupled dynamics between the angular momentum vector and the eccentricity vector as follows:

$$
\dot{\mathbf{e}}=a_{T} \Gamma \widehat{\mathbf{u}}=\frac{a_{T}}{\mu}[\widetilde{\mathbf{v}}][\widetilde{\mathbf{r}}] \widehat{\mathbf{u}}=\frac{1}{\mu}[\widetilde{\mathbf{v}}] \dot{\mathbf{h}} .
$$

For this reason, the angular momentum vector is sometimes decreased during the orbit transfer. That is, the semimajor axis is also decreased from the following relationship:

$$
\|\mathbf{h}\|=\sqrt{\mu p}=\sqrt{\mu a\left(1-e^{2}\right)},
$$

where $p \equiv a\left(1-e^{2}\right)$ is the semilatus rectum.

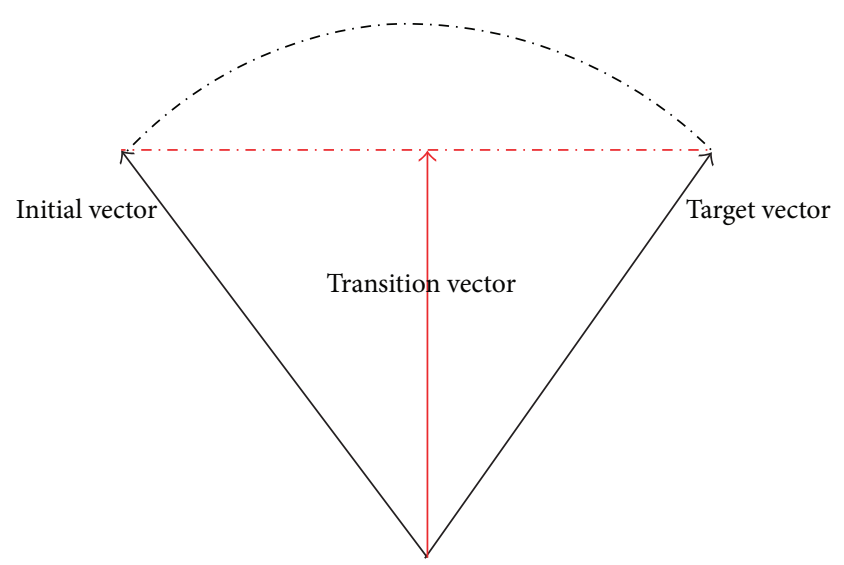

FIGURE 2: The conversion between classical orbital elements and vector notation.

The spacecraft model is described in Table 1 , and identity gain matrices are assumed.

Figure 3 shows the simulation result for the large-angle maneuver using the conditions described in Table 2. The total duration of orbit transfer is about 45.3 days by consuming the propellant fully. The initial and final eccentricities are the same; thus the semimajor axis is only affected by the angular momentum. Figure 3(a) shows that the altitude of the satellite is sometimes lower than the radius of the earth. That is, for the LEO, the satellite crashes with the earth by using this guidance scheme.

4.1. Guidance Scheme 1: Addition of an Energy Term. To avoid the crash problem for the large-angle maneuver using the previous guidance scheme, the semimajor axis of the transfer orbit must be controlled. A Lyapunov candidate adding the orbital energy term is proposed as follows:

$$
\begin{aligned}
V= & \frac{1}{2}\left(\mathbf{e}-\mathbf{e}_{f}\right)^{T} W_{1}\left(\mathbf{e}-\mathbf{e}_{f}\right) \\
& +\frac{1}{2} \frac{\left(\mathbf{h}-\mathbf{h}_{f}\right)^{T} W_{2}\left(\mathbf{h}-\mathbf{h}_{f}\right)[\widetilde{\mathbf{r}}]}{\left\|\mathbf{h}_{f}\right\|}+\frac{1}{2} k_{1} \frac{\left(E-E_{f}\right)^{2}}{E_{f}^{2}},
\end{aligned}
$$

where $E$ and $E_{f}$ are the orbital energy and the desired orbital energy, respectively. Note that the orbital energy term is normalized because this value is large. $k_{1}$ is the positive gain parameter. Thus, the Lyapunov candidate always has positive values except when the satellite achieved the desired orbit state. Therefore, during the orbit transfer, the time derivative of the Lyapunov candidate must have negative values to let the Lyapunov candidate go to zero.

The time derivative of the orbital energy is given by

$$
\dot{E}=a_{T} \mathbf{v}^{T} \widehat{\mathbf{u}} .
$$




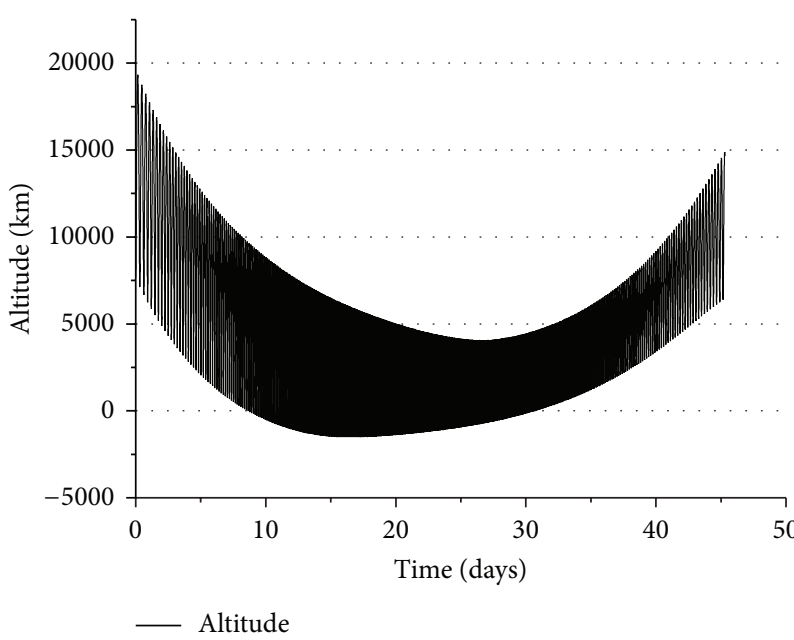

(a) The profile of the satellite altitude

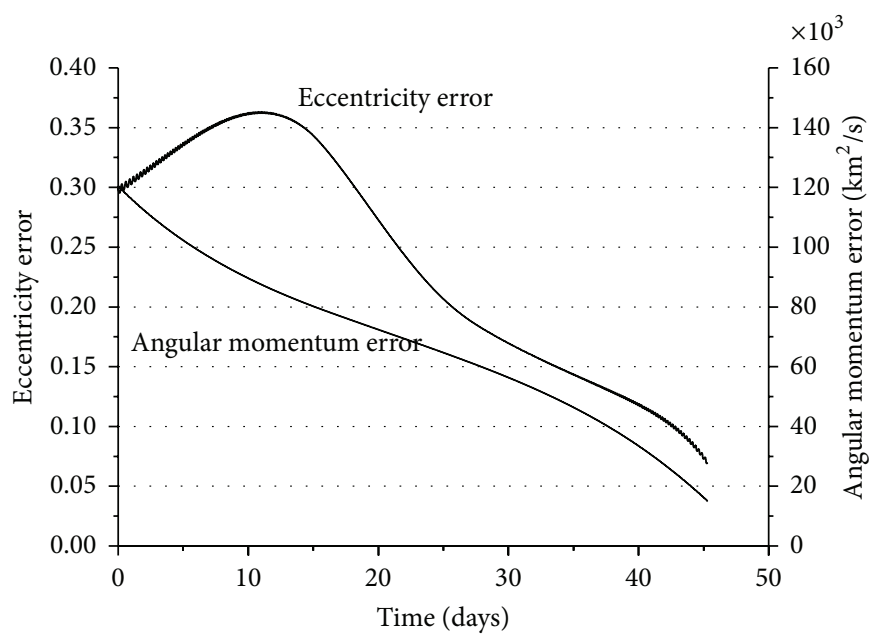

(b) The profile of the eccentricity vector error and the angular momentum vector error

FIGURE 3: The simulation result for the large-angle maneuver using the previous guidance scheme.

TABLE 1: The spacecraft model for the simulation.

\begin{tabular}{lc}
\hline Parts & Features \\
\hline Total mass for spacecraft (initial mass) & $350 \mathrm{~kg}$ \\
Total mass for propellant & $30 \%$ of the total \\
& mass for S/C \\
Thrust level & $1 \mathrm{~N}$ (ideal) \\
Specific impulse $\left(I_{\mathrm{sp}}\right)$ & $3800 \mathrm{sec}$ \\
\hline
\end{tabular}

TABLE 2: The first simulation conditions.

\begin{tabular}{lcc}
\hline & Initial values & $\begin{array}{c}\text { Desired } \\
\text { values }\end{array}$ \\
\hline Semimajor axis $(a)$ & $20000 \mathrm{~km}$ & $20000 \mathrm{~km}$ \\
Eccentricity $(e)$ & 0.3 & 0.3 \\
Inclination angle $(i)$ & $45^{\circ}$ & $45^{\circ}$ \\
$\begin{array}{l}\text { Longitude of the } \\
\text { ascending node }(\Omega)\end{array}$ & $205^{\circ}$ & $25^{\circ}$ \\
$\begin{array}{l}\text { Argument of perigee } \\
(\omega)\end{array}$ & $225^{\circ}$ & $45^{\circ}$ \\
\hline
\end{tabular}

Using (9) and (14), the time derivative of the Lyapunov candidate in (13) is written as

$$
\begin{aligned}
\frac{\mathrm{d} V}{\mathrm{~d} t}= & {\left[\left(\mathbf{e}-\mathbf{e}_{f}\right)^{T} W_{1} \Gamma+\frac{\left(\mathbf{h}-\mathbf{h}_{f}\right)^{T} W_{2}[\widetilde{\mathbf{r}}]}{\left\|\mathbf{h}_{f}\right\|}\right.} \\
& \left.+k_{1} \frac{E-E_{f}}{E_{f}^{2} \mathbf{v}^{T}}\right] a_{T} \widehat{\mathbf{u}}
\end{aligned}
$$

and $\widehat{\mathbf{u}}$ is selected to make this time derivative negative as follows:

$$
\begin{gathered}
\widehat{\mathbf{u}}=-\left(\left[\left(\mathbf{e}-\mathbf{e}_{f}\right)^{T} W_{1} \Gamma+\frac{\left(\mathbf{h}-\mathbf{h}_{f}\right)^{T} W_{2}[\widetilde{\mathbf{r}}]}{\left\|\mathbf{h}_{f}\right\|}\right.\right. \\
\left.+k_{1} \frac{E-E_{f}}{E_{f}^{2}} \mathbf{v}^{T}\right]^{T} \\
\quad \times\left(\|\left(\mathbf{e}-\mathbf{e}_{f}\right)^{T} W_{1} \Gamma \frac{\left(\mathbf{h}-\mathbf{h}_{f}\right)^{T} W_{2}[\widetilde{\mathbf{r}}]}{\left\|\mathbf{h}_{f}\right\|}\right. \\
\left.\left.+k_{1} \frac{E-E_{f}}{E_{f}^{2}} \mathbf{v}^{T} \|\right)^{-1}\right) .
\end{gathered}
$$

Now, (15) is always negative except when the orbit is the desired orbit or the thrust magnitude is zero.

Figure 4 shows the result for the large-angle maneuver using the conditions in Table 2 with guidance scheme 1 . The gain parameter $k_{1}=100$ is assumed for the soft constraint. Note that this value can be changed to improve the performance of the suggested guidance scheme. The total duration of orbit transfer is about 35.1 days and the crash problem from the previous orbital guidance scheme is successfully avoided.

This orbital guidance scheme has the redundancy term; initial and final orbit conditions are 5, and the Lyapunov candidate uses the six elements (eccentricity vector (3) + angular momentum vector (3) + orbital energy (1) - constraint between eccentricity vector and angular momentum vector (1)). For this reason, the transition vector of the angular momentum vector is affected by the orbital energy 


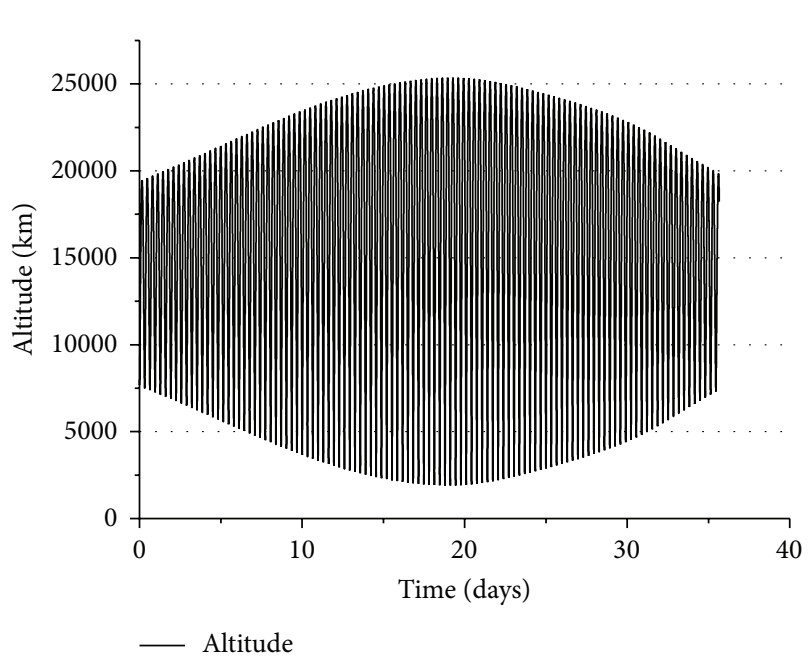

(a) The profile of the satellite altitude

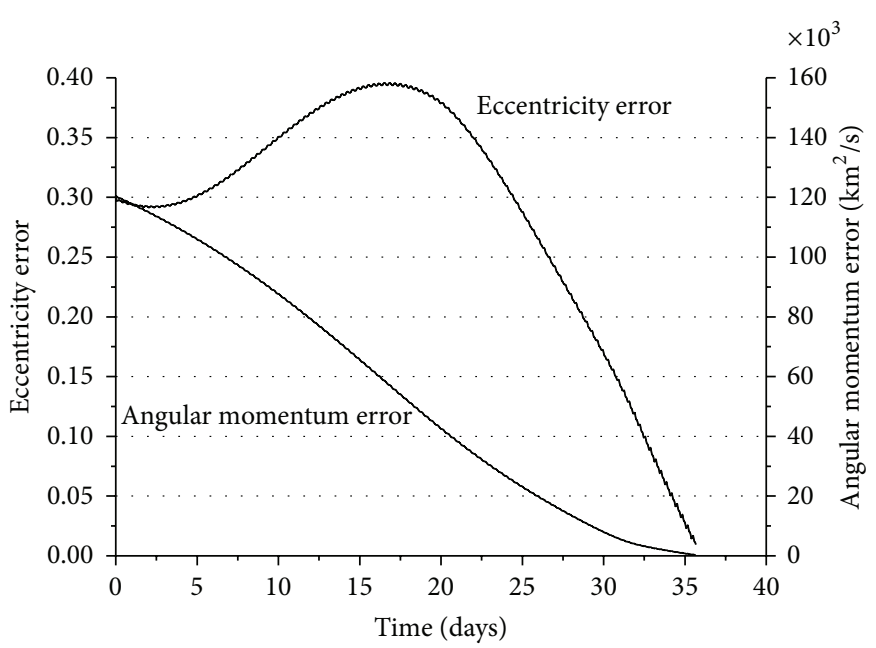

(b) The profile of the eccentricity vector error and the angular momentum vector error

FIGURE 4: The simulation result for the large-angle maneuver using the improved guidance scheme.

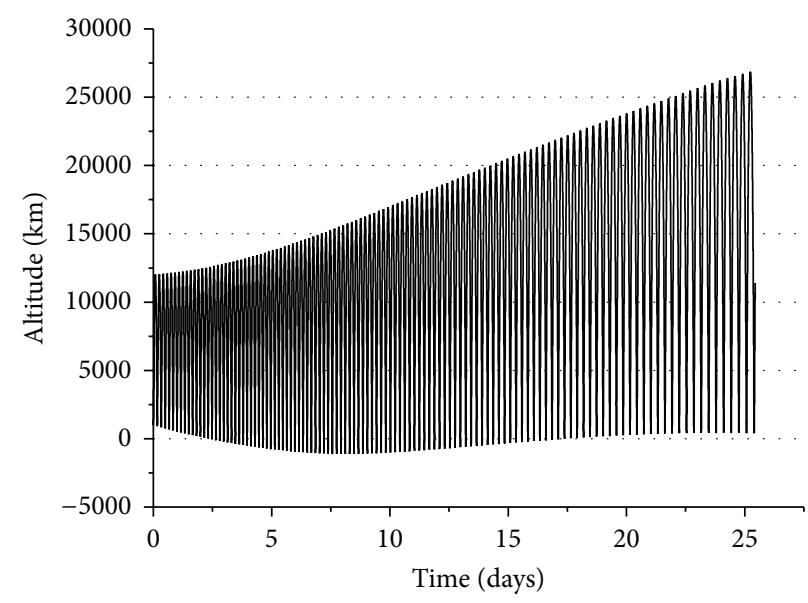

Altitude

(a) The profile of the satellite altitude

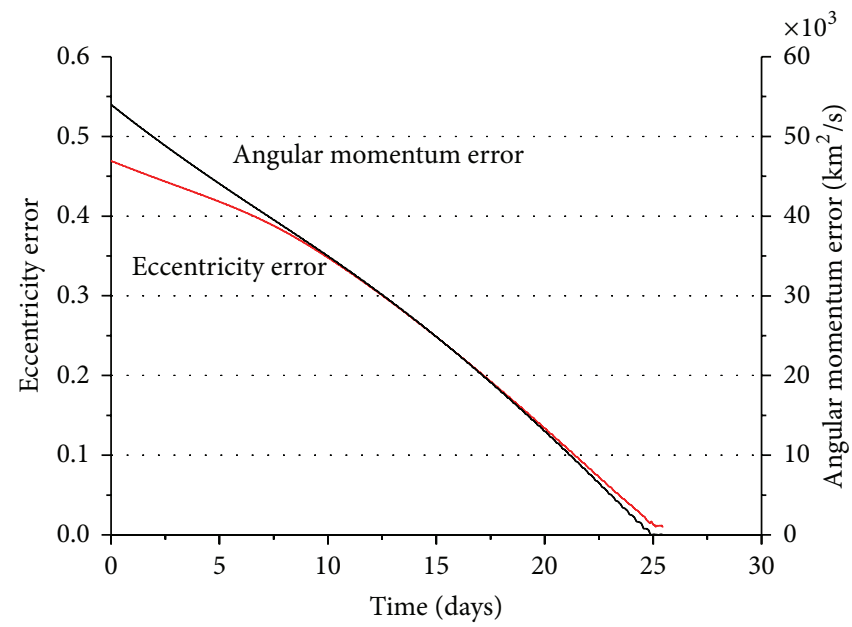

(b) The profile of the eccentricity vector error and the angular momentum vector error

FIGURE 5: The simulation result for the Molniya orbit using the improved guidance scheme.

term. Furthermore, the semimajor axis value is nearly under constraint. Therefore, the transition vector of the angular momentum vector is passing near the line between the initial and final angular momentum vectors. There is a difference between the transition vector and the line from the initial to the final angular momentum vector. This difference comes from the coupled dynamics between the angular momentum vector and the eccentricity vector as shown in (12).

Figure 5 shows the simulation result using the conditions in Table 3 with guidance scheme 1 . The gain matrices $W_{1}=$ $I_{3 \times 3}$ and $W_{2}=10 I_{3 \times 3}$ and the parameter $k_{1}=1$ are assumed. The initial orbit condition is as follows: the perigee and apogee altitude are $1,000 \mathrm{~km}$ and $12,000 \mathrm{~km}$, respectively, and the angles for the orientation are the arbitrary values. Then, at the final time, the semimajor and semiminor axes are $20,000 \mathrm{~km}$ and $15,000 \mathrm{~km}$, respectively. These final orbit states are similar to the Molniya orbit, which has very low altitude of the perigee. Therefore, one can find the transition orbit altitude using the previous guide scheme or guidance scheme 1. Figure 5(a) shows that the satellite passes the earth's surface (ground) or the low boundary altitude.

\subsection{Guidance Scheme 2: Addition of a Penalty Function Term.} To avoid the crash problem when the initial or final orbit state has the very low altitude of the perigee, the perigee of the transfer orbit must be considered as a constraint. For this 


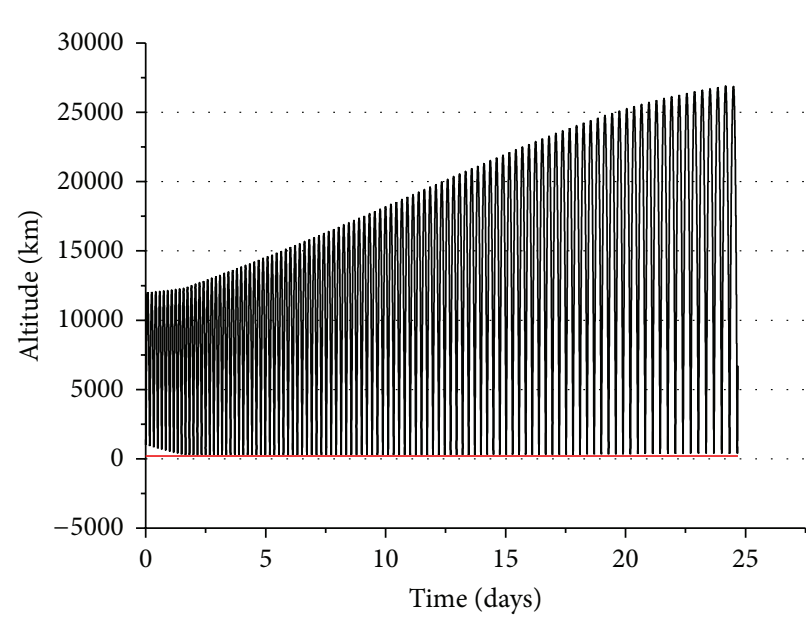

- Altitude

— Minimum bound

(a) The profile of the satellite altitude

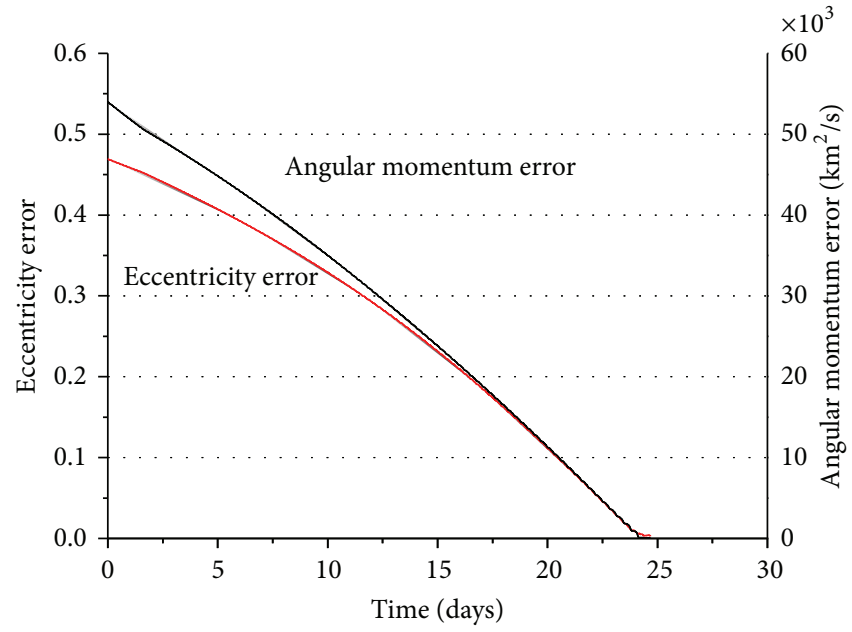

(b) The profile of the eccentricity vector error and the angular momentum vector error

FIGURE 6: The simulation result for the Molniya orbit using penalty function method.

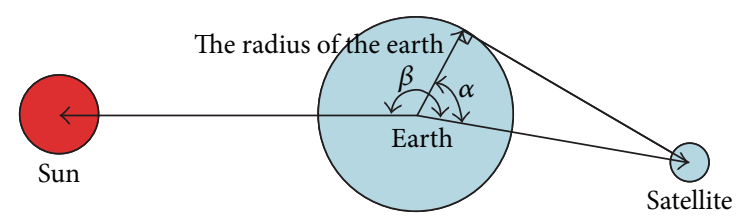

FIGURE 7: The calculation of the earth's shadow area.

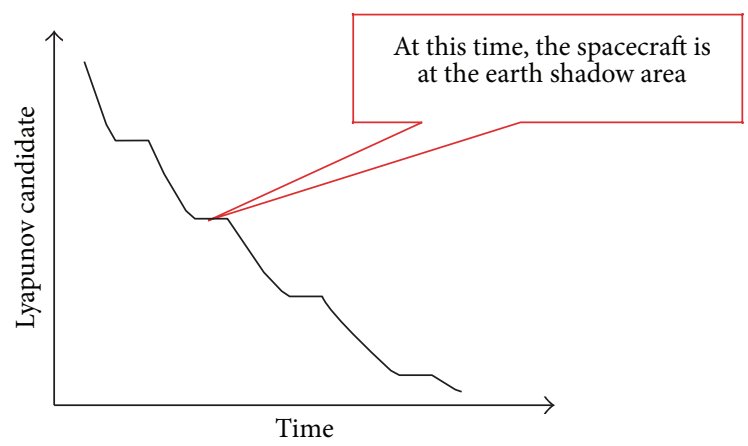

Figure 8: The Lyapunov candidate profile under the earth's shadow effect.

reason, a penalty function is suggested. To avoid the crash problem during the orbit transfer, it needs to be satisfied with the following condition:

$$
r_{p}=a(1-e) \geq r_{p c},
$$

where $r_{p}$ is the perigee radius and $r_{p c}$ is the minimum bound of the perigee radius. This condition can be rewritten as the following inequality condition:

$$
e \leq 1-\frac{r_{p c}}{a}=1+\frac{2 r_{p c} E}{\mu} .
$$

TABLE 3: The second simulation conditions.

\begin{tabular}{lcc}
\hline & Initial values & Desired values \\
\hline Semimajor axis $(a)$ & $12878.14 \mathrm{~km}$ & $20000 \mathrm{~km}$ \\
Eccentricity $(e)$ & 0.4270803 & 0.6614378 \\
$\begin{array}{l}\text { Inclination angle }(i) \\
\text { Longitude of the ascending } \\
\text { node }(\Omega)\end{array}$ & $20^{\circ}$ & $45^{\circ}$ \\
Argument of perigee $(\omega)$ & $90^{\circ}$ & $180^{\circ}$ \\
\hline
\end{tabular}

When the spacecraft is near the minimum bound of the perigee, the penalty function affects the orbital guidance scheme. In this work, the sigmoid function is used as a penalty function, which is expressed as

$$
s=\frac{1}{1+\exp (-c F)} .
$$

The shape of the sigmoid function can be changed by the positive constant value of $c$ and the function $F$. For example, if the value of $c$ is infinitely large, the sigmoid-function shape is similar to the step function. In addition, the sigmoid function has three values depending on the value of $F$ as follows: (i) $F<0 \rightarrow s=0$, (ii) $F>0 \rightarrow s=1$, and (iii) $F=0 \rightarrow s=1 / 2$.

From the sigmoid function in (19) and the inequality constraint in (18), $F$ is obtained as follows:

$$
F=\mathrm{e}^{2}-\left(1+\frac{2 r_{p c}}{\mu} E\right) .
$$




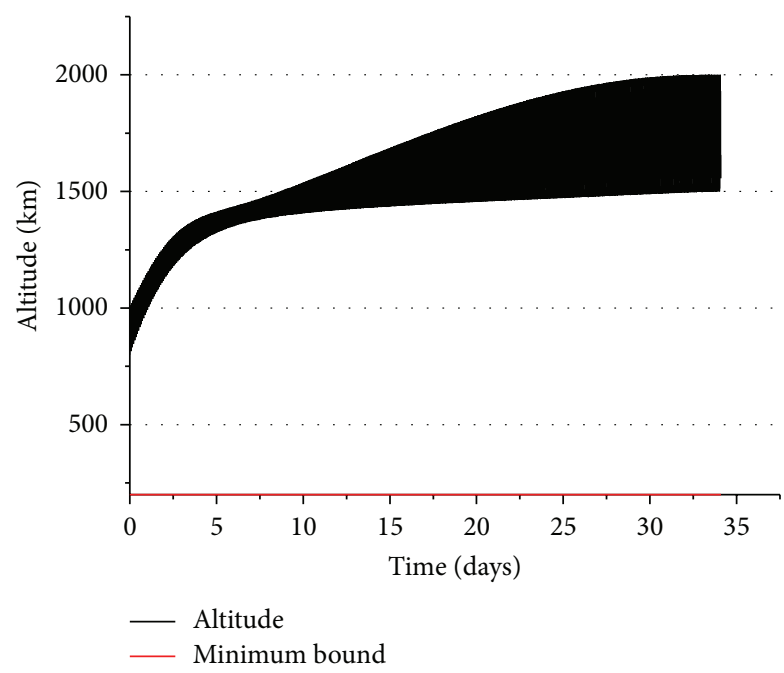

(a) The profile of the satellite altitude

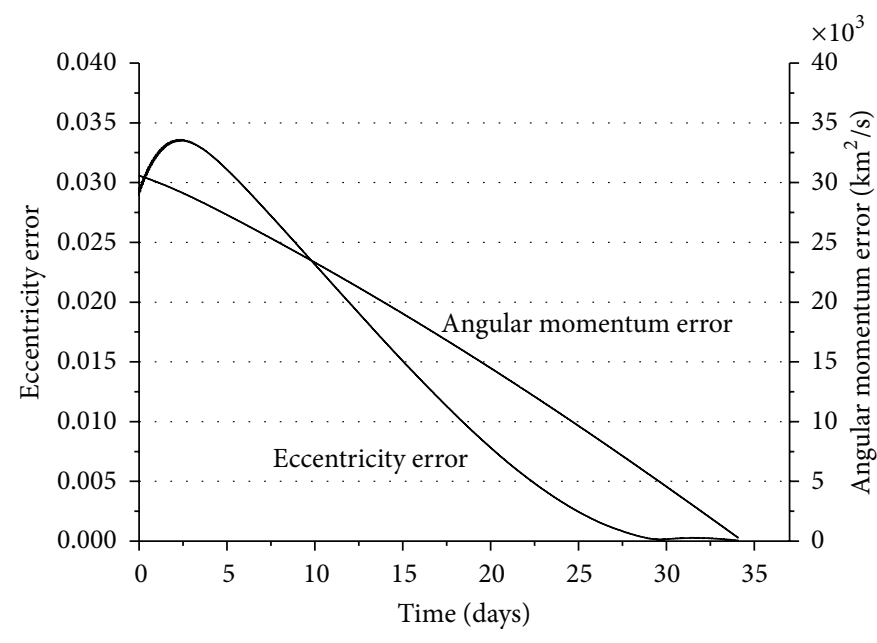

(b) The profile of the eccentricity vector error and the angular momentum vector error

FIgURE 9: The simulation result for the earth's shadow effect.

The penalty function is added to guidance scheme 1 , and the Lyapunov candidate is written as

$$
\begin{aligned}
V= & \frac{1}{2}\left(\mathbf{e}-\mathbf{e}_{f}\right)^{T} W_{1}\left(\mathbf{e}-\mathbf{e}_{f}\right)+\frac{1}{2} \frac{\left(\mathbf{h}-\mathbf{h}_{f}\right)^{T} W_{2}\left(\mathbf{h}-\mathbf{h}_{f}\right)[\widetilde{\mathbf{r}}]}{\left\|\mathbf{h}_{f}\right\|} \\
& +\frac{1}{2} k_{1} \frac{\left(E-E_{f}\right)^{2}}{E_{f}^{2}}+\frac{1}{2} k_{2} \frac{1}{1+\exp (-c F)},
\end{aligned}
$$

where $k_{2}$ is the positive gain parameter. Thus, the Lyapunov candidate always has positive values except when the satellite achieved the desired orbit state. Therefore, during the orbit transfer, the time derivative of the Lyapunov candidate must have negative values to let the Lyapunov candidate go to zero.

Using the chain rule, the time derivative of the penalty function is obtained as follows:

$$
\frac{\mathrm{d} s}{\mathrm{~d} t}=\frac{\mathrm{d} s}{\mathrm{~d} F} \frac{\mathrm{d} F}{\mathrm{~d} t}=2 \Delta a_{T} \widehat{\mathbf{u}}
$$

where $\Delta$ is defined as

$$
\begin{aligned}
\Delta= & {\left[\frac{c}{\exp (c F)+2+\exp (-c F)}\right] } \\
& \times\left[\mathbf{e}^{T} \Gamma-\left(1+\frac{2 r_{p c}}{\mu} E\right) \frac{2 r_{p c}}{\mu} \mathbf{v}^{T}\right] .
\end{aligned}
$$

Using (15) and (22), the time derivative of the Lyapunov candidate in (21) is written as

$$
\begin{gathered}
\frac{\mathrm{d} V}{\mathrm{~d} t}=\left[\left(\mathbf{e}-\mathbf{e}_{f}\right)^{T} W_{1} \Gamma+\frac{\left(\mathbf{h}-\mathbf{h}_{f}\right)^{T} W_{2}[\widetilde{\mathbf{r}}]}{\left\|\mathbf{h}_{f}\right\|}\right. \\
\left.+k_{1} \frac{E-E_{f}}{E_{f}^{2}} \mathbf{v}^{T}+k_{2} \Delta\right] a_{T} \widehat{\mathbf{u}}
\end{gathered}
$$

and $\widehat{\mathbf{u}}$ is selected to make this time derivative negative as follows:

$$
\begin{array}{r}
\widehat{\mathbf{u}}=-\left(\left[\left(\mathbf{e}-\mathbf{e}_{f}\right)^{T} W_{1} \Gamma+\frac{\left(\mathbf{h}-\mathbf{h}_{f}\right)^{T} W_{2}[\widetilde{\mathbf{r}}]}{\left\|\mathbf{h}_{f}\right\|}\right.\right. \\
\left.+k_{1} \frac{E-E_{f}}{E_{f}^{2}} \mathbf{v}^{T}+k_{2} \Delta\right]^{T} \\
\times\left(\|\left(\mathbf{e}-\mathbf{e}_{f}\right)^{T} W_{1} \Gamma+\frac{\left(\mathbf{h}-\mathbf{h}_{f}\right)^{T} W_{2}[\widetilde{\mathbf{r}}]}{\left\|\mathbf{h}_{f}\right\|}\right. \\
\left.\left.+k_{1} \frac{E-E_{f}}{E_{f}^{2}} \mathbf{v}^{T}+k_{2} \Delta \|\right)^{-1}\right) .
\end{array}
$$

Now, (24) is always negative except when the orbit is the desired orbit or the thrust magnitude is zero.

Figure 6 shows the simulation result using the conditions in Table 3 with guidance scheme 2. The gain parameter 


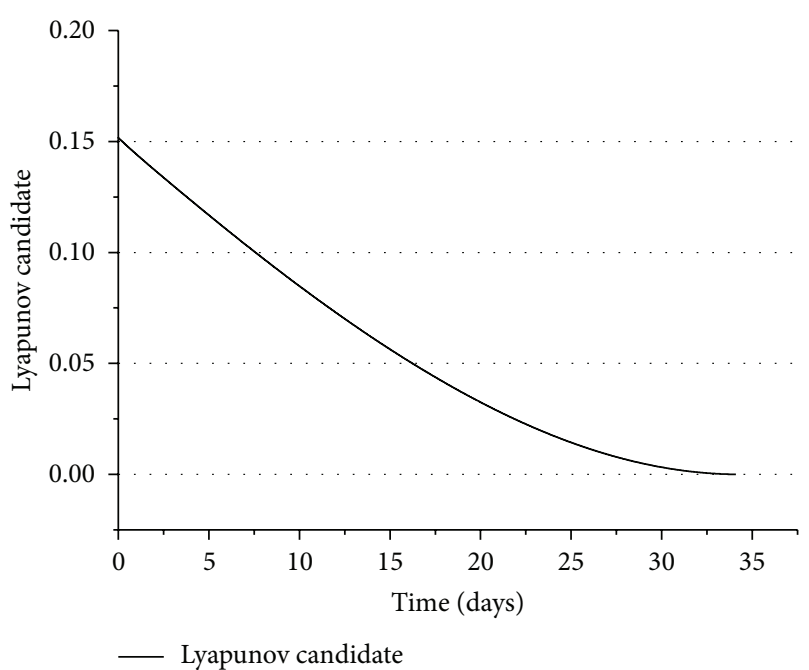

(a)

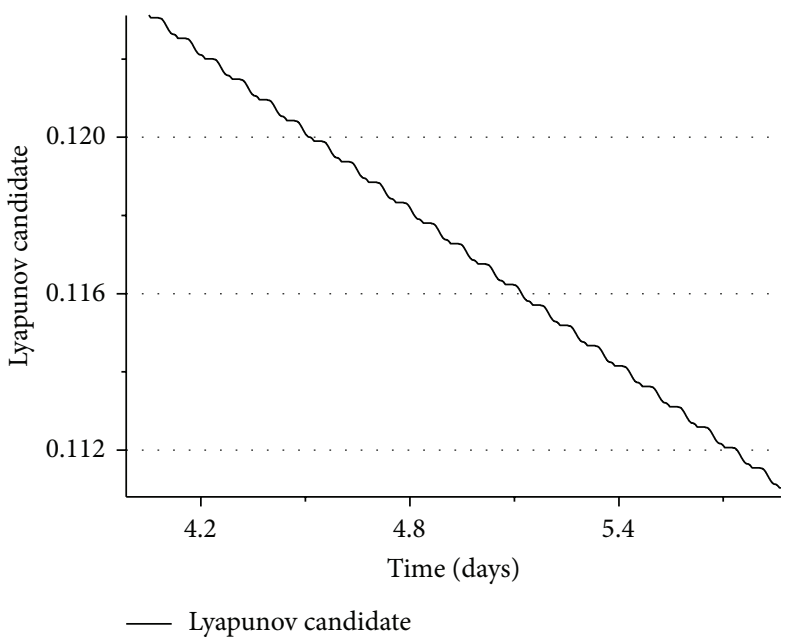

(b)

FIGURE 10: The profile of the Lyapunov candidate under the earth's shadow effect.

$k_{2}=1$ is assumed. The total duration of orbit transfer is about 24.7 days. As shown in Figure 6(a), the crash problem is completely avoided by considering the penalty function, which affects the orbit state. Now, the semimajor axis value is under constraint, and the altitude of the perigee is bounded. Regardless of the increase of the consumed mass of the satellite, the reliability is guaranteed for the orbit transfer mission.

\section{Applied Earth's Shadow Effect}

Several types of the orbital guidance schemes are discussed in previous sections. The guidance command is generated to make negative values for the derivative of the Lyapunov candidate, and the derivative of the Lyapunov candidate is zero when the magnitude of the thrust acceleration is zero. This means that the electrical propulsion system is not operated, and one of operating modes is the eclipse mode. Consider small satellite missions on LEOs, especially when a satellite is in the earth's shadow area. During this time, supplying electrical powers to the satellite is going to be difficult, because of the insufficient sun power for the solar array. For this reason, the thrust magnitude is zero during this time. Figure 7 shows the position of the satellite and the sun for the earth's shadow area. When the angle $\beta$ between the sun and the satellite is larger than the sum of the angle $\alpha$, which is the half cone angle, and $90^{\circ}$, the satellite is under the earth's shadow area. That is, the earth's shadow effect is applied when the following condition is satisfied:

$$
\beta>\alpha+90^{\circ} .
$$

In the earth's shadow area shown in Figure 8, the thrust magnitude is zero, and the derivative of Lyapunov candidate is the seminegative definite. In general, when the derivative of Lyapunov candidate is the seminegative definite, the Lyapunov candidate is not always converged into zero. Since
TABLE 4: The third simulation conditions.

\begin{tabular}{lcc}
\hline & Initial values & Desired values \\
\hline Semimajor axis $(a)$ & $7278.14 \mathrm{~km}$ & $7478.14 \mathrm{~km}$ \\
Eccentricity $(e)$ & 0.0137398 & 0.0133723 \\
$\begin{array}{l}\text { Inclination angle }(i) \\
\text { Longitude of the ascending } \\
\text { node }(\Omega)\end{array}$ & $15^{\circ}$ & $45^{\circ}$ \\
Argument of perigee $(\omega)$ & $25^{\circ}$ & $0^{\circ}$ \\
\hline
\end{tabular}

the spacecraft is always turning around the earth, however, the sum of the derivative of the Lyapunov candidate during one revolution is always the negative definite. Thus, the Lyapunov candidate is always converged into zero for the orbit transfer in elliptic orbits. This means that the desired orbital conditions can be approached successfully.

Figures 9 and 10 show the simulation result using the conditions in Table 4 with guidance scheme 2. For simplicity, a fixed position for the sun and the position vector $1 A U \times$ $[e, 0,0]^{T}$ are assumed. The total duration of orbit transfer is about 14.8 days. Figure 10(b) shows that the Lyapunov candidate values are not changed when the satellite is passing the earth's shadow area.

From these results, we show that guidance scheme 2 can be applied on the satellite passing the earth's shadow area.

\section{Conclusions}

In this study, for the simplicity and avoidance of the singularity of the orbit dynamics, the vector notation is utilized to describe the motion of the satellite moving around the earth's gravity field. The Lyapunov feedback control method is used as a simple and reliable guidance scheme with the vector notation. In the previous study, the eccentricity and angular 
momentum vectors are considered as a Lyapunov candidate to avoid singularities of the orbit dynamics. However, the altitude of the satellite is sometimes lower than the surface of the earth using the previous guidance scheme. To avoid the crash problem, a vector constraint is considered by using the energy term in the Lyapunov candidate. The soft constraint about the altitude is considered, and the crash problem is avoided for the large-angle maneuver. Furthermore, the crash problem including the Molniya orbit is completely avoided by using the penalty function. In addition, the orbit transfer under the earth's shadow effect is also successfully achieved by using guidance scheme 2 , which is the general one and would be very useful for solving any orbit transfer problems.

\section{Conflict of Interests}

The authors declare that there is no conflict of interests regarding the publication of this paper.

\section{Acknowledgment}

This research was supported by the project "A Development of Space Debris Collision Management System and a Space on the Removal System," which is a part of "National Agenda Program: Development of Electro-Optic Space Surveillance System" through the Korea Research Council Fundamental Science \& Technology.

\section{References}

[1] M. Guelman, "Earth-to-moon transfer with a limited power engine," Journal of Guidance, Control, and Dynamics, vol. 18, no. 5, pp. 1133-1138, 1995.

[2] B. L. Pierson and C. A. Kluever, "Three-stage approach to optimal low-thrust earth-moon trajectories," Journal of Guidance, Control, and Dynamics, vol. 17, no. 6, pp. 1275-1282, 1994.

[3] P. J. Enright and B. A. Conway, "Optimal finite-thrust spacecraft trajectories using collocation and nonlinear programming," Journal of Guidance, Control, and Dynamics, vol. 14, no. 5, pp. 981-985, 1991.

[4] J. T. Betts, "Very low-thrust trajectory optimization using a direct SQP method," Journal of Computational and Applied Mathematics, vol. 120, no. 1-2, pp. 27-40, 2000.

[5] M. Hawkins, Y. Guo, and B. Wie, "Spacecraft guidance algorithms for asteroid intercept and rendezvous missions," International Journal of Aeronautical and Space Sciences, vol. 13, no. 2, pp. 154-169, 2012.

[6] Y. Gao and C. A. Kluever, "Low-thrust guidance scheme for earth-capture trajectories," Journal of Guidance, Control, and Dynamics, vol. 28, no. 2, pp. 333-342, 2005.

[7] C. A. Kluever, "Optimal earth-capture trajectories using electric propulsion," Journal of Guidance, Control, and Dynamics, vol. 25 , no. 3, pp. 604-606, 2002.

[8] C. A. Kluever, "Simple guidance scheme for low-thrust orbit transfers," Journal of Guidance, Control, and Dynamics, vol. 21, no. 6, pp. 1015-1017, 1998.

[9] M. R. Ilgen, "Low thrust OTV guidance using Liapunov optimal feedback control techniques," Advances in the Astronautical Sciences, vol. 85, no. 2, pp. 1527-1545, 1993, AAS 93-680, 1993.
[10] A. E. Petropoulos, "Low-thrust orbit transfers using candidate lyapunov functions with a mechanism for coasting," in AAS/AIAA Astrodynamics Specialist Conference, August 2004, AAS Paper 04-5089.

[11] A. E. Petropoulos, "Refinements to the Q-law for low-thrust orbit transfers," Advances in the Astronautical Sciences, vol. 120, no. 1, pp. 963-982, 2005.

[12] S. R. Vadali, K. Aroonwilairut, and E. Braden, "A hybrid trajectory optimization technique for the mars sample return mission," Advances in the Astronautical Sciences, vol. 109, pp. 2309-2320, 2002.

[13] D. E. Chang, D. F. Chichka, and J. E. Marsden, "Lyapunovbased transfer between elliptic Keplerian orbits," Discrete and Continuous Dynamical Systems B, vol. 2, no. 1, pp. 57-67, 2002.

[14] S. Hernandez and M. R. Akella, "Lyapunov-based guidance for orbit transfers and rendezvous in Levi-Civita coordinates," Journal of Guidance, Control, and Dynamics, 2014.

[15] J. A. Kéchichian, "Constrained circularization in elliptic orbit using low thrust with shadowing effect," Journal of Guidance, Control, and Dynamics, vol. 26, no. 6, pp. 949-955, 2003.

[16] D.-H. Cho, D.-H. Lee, and M.-J. Tahk, "Orbit transfer trajectory optimization with electric engine," in Proceeding of the SICEICASE International Joint Conference, pp. 4093-4098, Busan, Republic of Korea, October 2006.

[17] H. Schaub and J. L. Junkins, Analytical Mechanics of Space Systems, AIAA Education Series, 2009. 


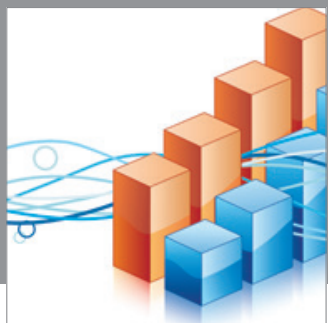

Advances in

Operations Research

mansans

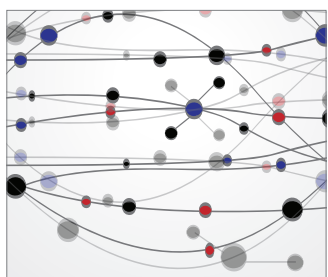

The Scientific World Journal
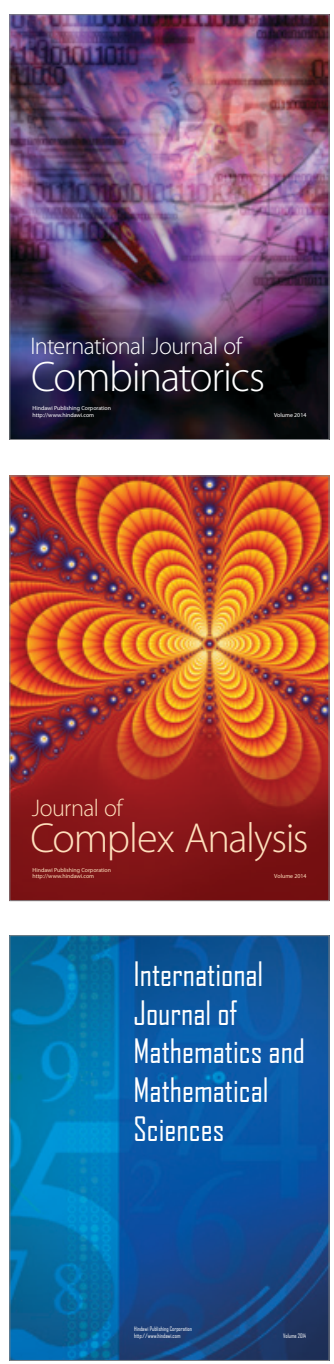
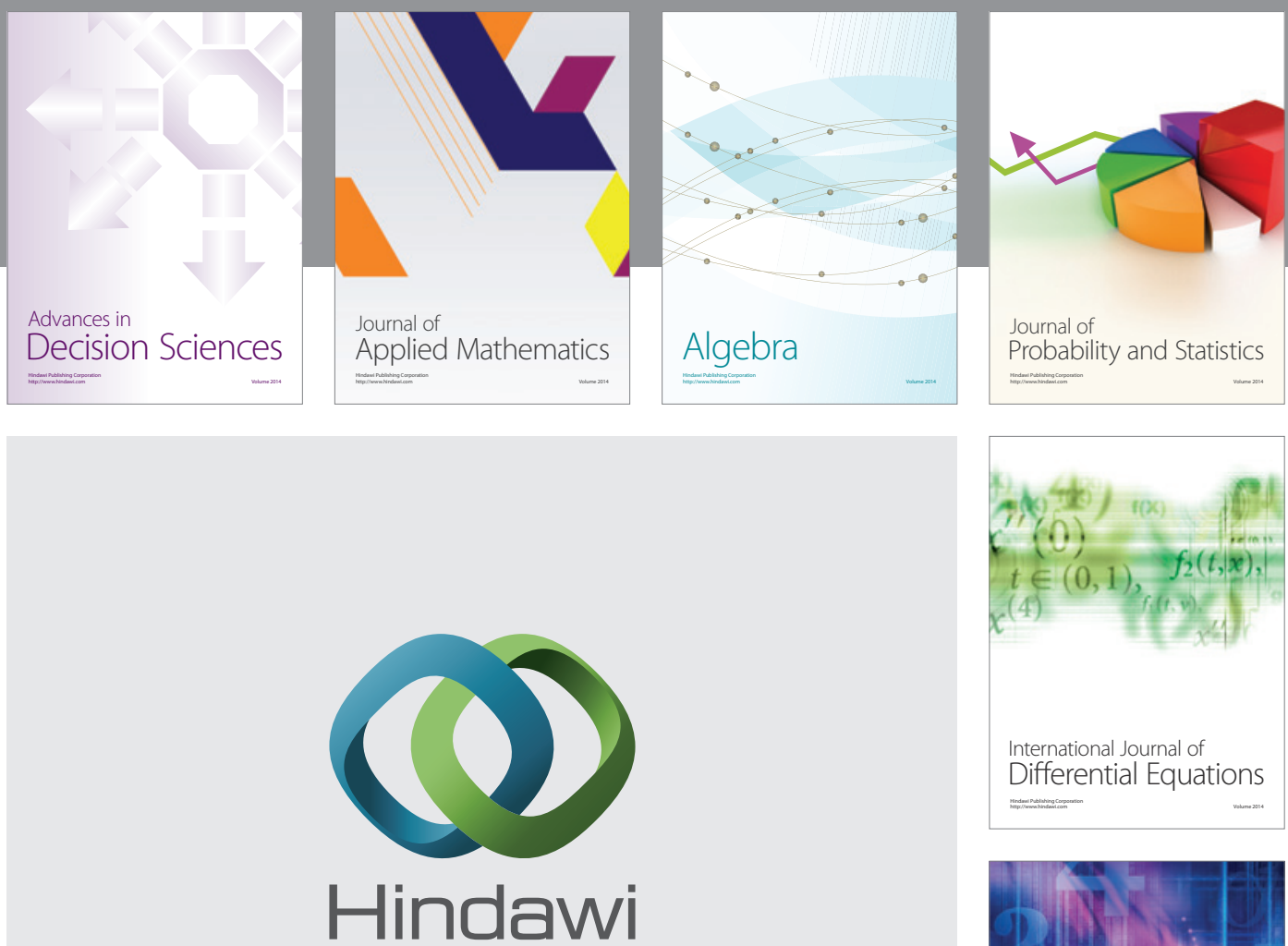

Submit your manuscripts at http://www.hindawi.com
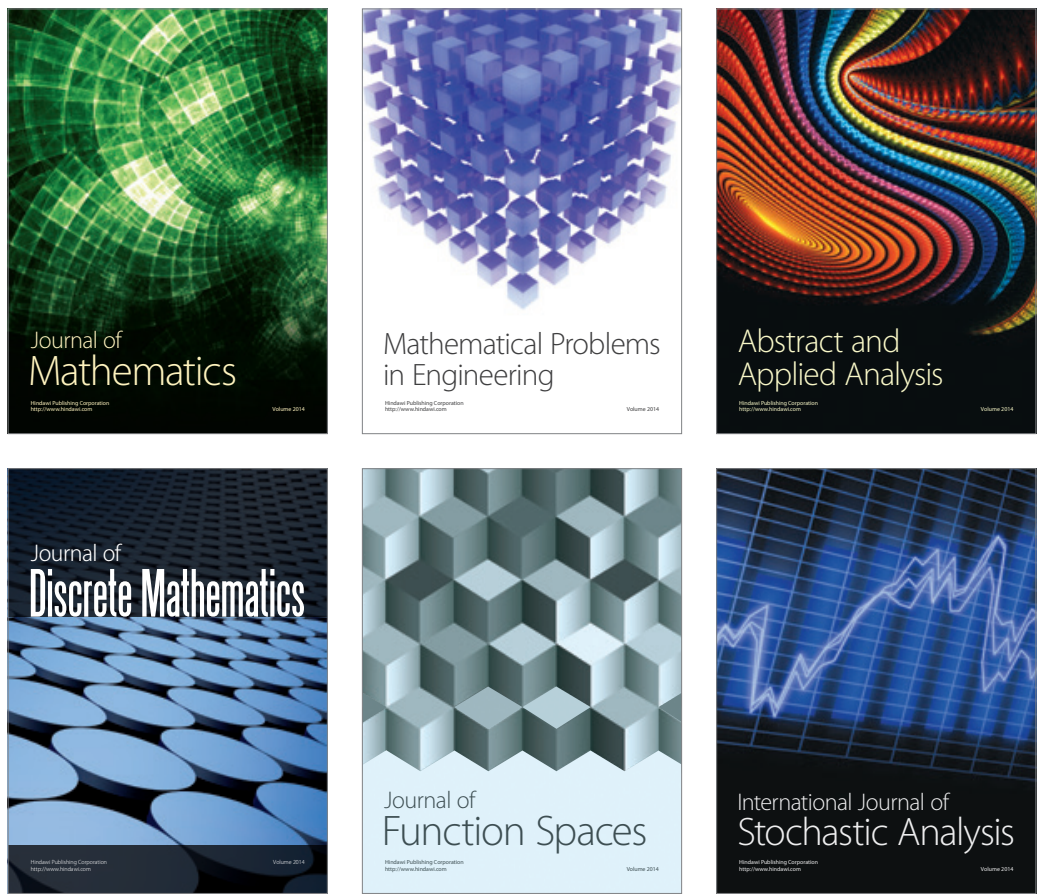

Journal of

Function Spaces

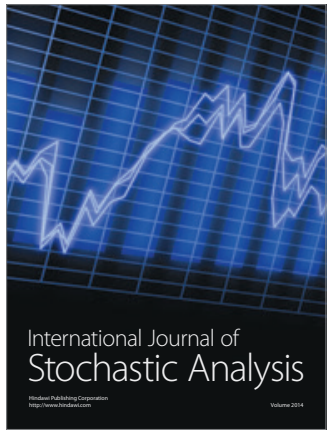

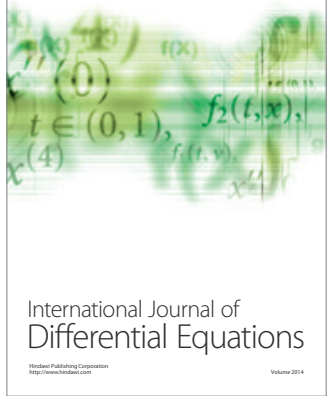
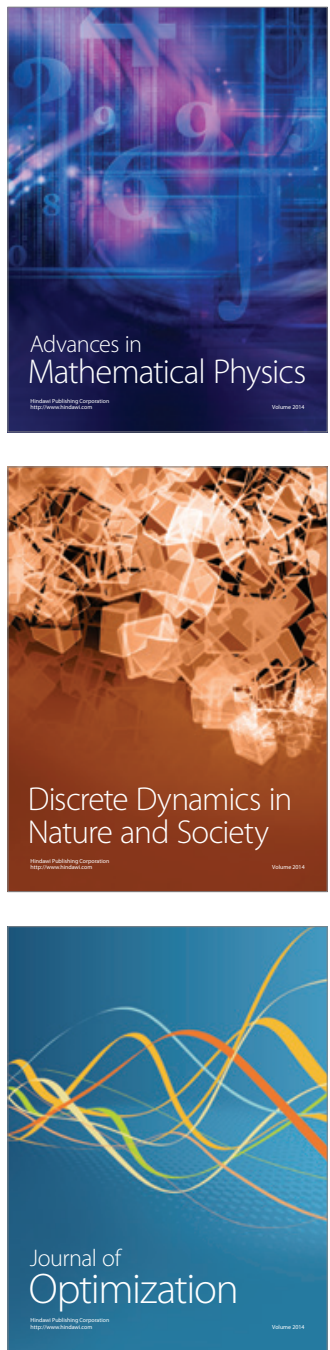